\title{
Parabolic Anderson model with rough dependence in space
}

\author{
Yaozhong Hu*, Jingyu Huang, Khoa Lê, David Nualart† Samy Tindel ${ }^{\ddagger}$
}

\begin{abstract}
This paper studies the one-dimensional parabolic Anderson model driven by a Gaussian noise which is white in time and has the covariance of a fractional Brownian motion with Hurst parameter $H \in\left(\frac{1}{4}, \frac{1}{2}\right)$ in the space variable. We derive the Wiener chaos expansion of the solution and a Feynman-Kac formula for the moments of the solution. These results allow us to establish sharp lower and upper asymptotic bounds for the $n$th moment of the solution.

Keywords. Stochastic heat equation, fractional Brownian motion, Feynman-Kac formula, Wiener chaos expansion, sharp lower and upper moment bounds, intermittency AMS 2010 subject classification. Primary 60H15; Secondary 35R60, 60G60.
\end{abstract}

\section{Introduction}

A recent paper [9] studies the stochastic heat equation for $(t, x) \in(0, \infty) \times \mathbb{R}$

$$
\frac{\partial u}{\partial t}=\frac{\kappa}{2} \frac{\partial^{2} u}{\partial x^{2}}+\sigma(u) \dot{W},
$$

where $\dot{W}$ is a centered Gaussian noise which is white in time and behaves as fractional Brownian motion with Hurst parameter $1 / 4<H<1 / 2$ in space, and $\sigma$ may be a nonlinear function with some smoothness.

However, the specific case $\sigma(u)=u$, i.e.

$$
\frac{\partial u}{\partial t}=\frac{\kappa}{2} \frac{\partial^{2} u}{\partial x^{2}}+u \dot{W}
$$

deserves some specific treatment due to its simplicity. Indeed, this linear equation turns out to be a continuous version of the parabolic Anderson model, and is related to challenging systems in random environment like $\mathrm{KPZ}$ equation $[6,3]$ or polymers $[1,4]$. The localization and intermittency properties (1.2) have thus been thoroughly studied for equations driven by a space-time white noise (see [14] for a nice survey), while a recent trend consists in extending this kind of result to equations driven by very general Gaussian noises $[5,10,8,11]$, but the rough noise $\dot{W}$ presented here is not covered by the aforementioned references.

\footnotetext{
${ }^{*}$ Y. Hu is partially supported by a grant from the Simons Foundation \#209206.

${ }^{\dagger}$ D. Nualart is supported by the NSF grant DMS1208625 and the ARO grant FED0070445.

${ }^{\ddagger}$ S. Tindel is supported by the NSF grant DMS1613163
} 
To fill this gap, we first go to the existence and uniqueness problem. Although the existence and uniqueness of the solution in the general nonlinear case (1.1) has been established in [9], in this linear case (1.2), one can implement a rather simple procedure involving Fourier transforms. Since this point of view is interesting in its own right and is short enough, we develop it in Subsection 3.1. In Subsection 3.2, we study the random field solution using chaos expansion. Following the approach introduced in $[8,10]$, we obtain an explicit formula for the kernels of the Wiener chaos expansion and we show its convergence, and thus obtain the existence and uniqueness of the solution. It worths noting these methods treat different classes of initial data which are more general than in [9] and different from [2].

We then move to a Feynman-Kac type representation for the moments of the solution. In fact, we cannot expect a Feynman-Kac formula for the solution, because the covariance is rougher than the space-time white noise case, and this type of formula requires smoother covariance structures (see, for instance, [11]). However, by means of Fourier analysis techniques as in $[8,10]$, we are able to obtain a Feynman-Kac formula for the moments that involves a fractional derivative of the Brownian local time.

Finally, the previous considerations allow us to handle, in the last section of the paper, the intermittency properties of the solution. More precisely, we show sharp lower bounds for the moments of the solution of the form $\mathbf{E}\left[u(t, x)^{n}\right] \geq \exp \left(C n^{1+\frac{1}{H}} t\right)$, for all $t \geq 0, x \in \mathbb{R}$ and $n \geq 2$, where $C$ is independent of $t \geq 0, x \in \mathbb{R}$ and $n$. These bounds entail the intermittency phenomenon and match the corresponding estimates for the case $H>\frac{1}{2}$ obtained in [10]. After the completion of this work, three of the authors have studied the parabolic Anderson model in more details in [12]. In particular, existence and uniqueness results are extended for a wider class of initial data, exact long term asymptotics for the moments of the solution are obtained.

\section{Preliminaries}

Let us start by introducing our basic notation on Fourier transforms of functions. The space of Schwartz functions is denoted by $\mathcal{S}$. Its dual, the space of tempered distributions, is $\mathcal{S}^{\prime}$. The Fourier transform of a function $u \in \mathcal{S}$ is defined with the normalization

$$
\mathcal{F} u(\xi)=\int_{\mathbb{R}} e^{-i \xi x} u(x) d x
$$

so that the inverse Fourier transform is given by $\mathcal{F}^{-1} u(\xi)=(2 \pi)^{-1} \mathcal{F} u(-\xi)$.

Let $\mathcal{D}((0, \infty) \times \mathbb{R})$ denote the space of real-valued infinitely differentiable functions with compact support on $(0, \infty) \times \mathbb{R}$. Taking into account the spectral representation of the covariance function of the fractional Brownian motion in the case $H<\frac{1}{2}$ proved in [17, Theorem 3.1], we represent our noise $W$ by a zero-mean Gaussian family $\{W(\varphi), \varphi \in \mathcal{D}((0, \infty) \times \mathbb{R})\}$ defined on a complete probability space $(\Omega, \mathcal{F}, \mathbf{P})$, whose covariance structure is given by

$$
\mathbf{E}[W(\varphi) W(\psi)]=c_{1, H} \int_{\mathbb{R}_{+} \times \mathbb{R}} \mathcal{F} \varphi(s, \xi) \overline{\mathcal{F} \psi(s, \xi)}|\xi|^{1-2 H} d s d \xi
$$

where the Fourier transforms $\mathcal{F} \varphi, \mathcal{F} \psi$ are understood as Fourier transforms in space only and

$$
c_{1, H}=\frac{1}{2 \pi} \Gamma(2 H+1) \sin (\pi H) .
$$


We denote by $\mathfrak{H}$ the Hilbert space obtained by completion of $\mathcal{D}((0, \infty) \times \mathbb{R})$ with respect to the inner product

$$
\langle\varphi, \psi\rangle_{\mathfrak{H}}=c_{1, H} \int_{\mathbb{R}_{+} \times \mathbb{R}} \mathcal{F} \varphi(s, \xi) \overline{\mathcal{F} \psi(s, \xi)}|\xi|^{1-2 H} d \xi d s
$$

The next proposition is from Theorem 3.1 and Proposition 3.4 in [17].

Proposition 2.1. If $\mathfrak{H}_{0}$ denotes the class of functions $\varphi \in L^{2}\left(\mathbb{R}_{+} \times \mathbb{R}\right)$ such that

$$
\int_{\mathbb{R}_{+} \times \mathbb{R}}|\mathcal{F} \varphi(s, \xi)|^{2}|\xi|^{1-2 H} d \xi d s<\infty
$$

then $\mathfrak{H}_{0}$ is not complete and the inclusion $\mathfrak{H}_{0} \subset \mathfrak{H}$ is strict.

We recall that the Gaussian family $W$ can be extended to $\mathfrak{H}$ and this produces an isonormal Gaussian process, for which Malliavin calculus can be applied. We refer to [16] and [7] for a detailed account of the Malliavin calculus with respect to a Gaussian process. On our Gaussian space, the smooth and cylindrical random variables $F$ are of the form

$$
F=f\left(W\left(\phi_{1}\right), \ldots, W\left(\phi_{n}\right)\right)
$$

with $\phi_{i} \in \mathfrak{H}, f \in C_{p}^{\infty}\left(\mathbb{R}^{n}\right)$ (namely $f$ and all its partial derivatives have polynomial growth). For this kind of random variable, the derivative operator $D$ in the sense of Malliavin calculus is the $\mathfrak{H}$-valued random variable defined by

$$
D F=\sum_{j=1}^{n} \frac{\partial f}{\partial x_{j}}\left(W\left(\phi_{1}\right), \ldots, W\left(\phi_{n}\right)\right) \phi_{j} .
$$

The operator $D$ is closable from $L^{2}(\Omega)$ into $L^{2}(\Omega ; \mathfrak{H})$ and we define the Sobolev space $\mathbb{D}^{1,2}$ as the closure of the space of smooth and cylindrical random variables under the norm

$$
\|D F\|_{1,2}=\sqrt{\mathbf{E}\left[F^{2}\right]+\mathbf{E}\left[\|D F\|_{\mathfrak{H}}^{2}\right]} .
$$

We denote by $\delta$ the adjoint of the derivative operator (called divergence operator) given by the duality formula

$$
\mathbf{E}[\delta(u) F]=\mathbf{E}\left[\langle D F, u\rangle_{\mathfrak{H}}\right],
$$

for any $F \in \mathbb{D}^{1,2}$ and any element $u \in L^{2}(\Omega ; \mathfrak{H})$ in the domain of $\delta$.

For any integer $n \geq 0$ we denote by $\mathbf{H}_{n}$ the $n$th Wiener chaos of $W$. We recall that $\mathbf{H}_{0}$ is simply $\mathbb{R}$ and for $n \geq 1, \mathbf{H}_{n}$ is the closed linear subspace of $L^{2}(\Omega)$ generated by the random variables $\left\{H_{n}(W(\phi)), \phi \in \mathfrak{H},\|\phi\|_{\mathfrak{H}}=1\right\}$, where $H_{n}$ is the $n$th Hermite polynomial. For any $n \geq 1$, we denote by $\mathfrak{H}^{\otimes n}$ (resp. $\mathfrak{H}^{\odot n}$ ) the $n$th tensor product (resp. the $n$th symmetric tensor product) of $\mathfrak{H}$. Then, the mapping $I_{n}\left(\phi^{\otimes n}\right)=H_{n}(W(\phi))$ can be extended to a linear isometry between $\mathfrak{H}^{\odot n}$ (equipped with the modified norm $\sqrt{n !}\|\cdot\|_{\mathfrak{H}^{\otimes n}}$ ) and $\mathbf{H}_{n}$.

Consider now a random variable $F \in L^{2}(\Omega)$ which is measurable with respect to the $\sigma$-field $\mathcal{F}$ generated by $W$. This random variable can be expressed as

$$
F=\mathbf{E}[F]+\sum_{n=1}^{\infty} I_{n}\left(f_{n}\right)
$$


where the series converges in $L^{2}(\Omega)$, and the elements $f_{n} \in \mathfrak{H}^{\odot n}, n \geq 1$, are determined by $F$. This identity is called the Wiener chaos expansion of $F$.

The Skorohod integral (or divergence) of a random field $u$ can be computed by using the Wiener chaos expansion. More precisely, suppose that $u=\left\{u(t, x),(t, x) \in \mathbb{R}_{+} \times \mathbb{R}\right\}$ is a random field such that for each $(t, x), u(t, x)$ is an $\mathcal{F}_{t}$-measurable and square-integrable random variable, here $\mathcal{F}_{t}$ is the $\sigma$ algebra generated by $W$ up to time $t$. Then, for each $(t, x)$ we have a Wiener chaos expansion of the form

$$
u(t, x)=\mathbf{E}[u(t, x)]+\sum_{n=1}^{\infty} I_{n}\left(f_{n}(\cdot, t, x)\right) .
$$

Suppose that $\mathbf{E}\left[\|u\|_{\mathfrak{H}}^{2}\right]$ is finite. Then, we can interpret $u$ as a square-integrable random function with values in $\mathfrak{H}$ and the kernels $f_{n}$ in the expansion (2.6) are functions in $\mathfrak{H}^{\otimes(n+1)}$ which are symmetric in the first $n$ variables. In this situation, $u$ belongs to the domain of the divergence operator (that is, $u$ is Skorohod integrable with respect to $W$ ) if and only if the following series converges in $L^{2}(\Omega)$

$$
\delta(u)=\int_{0}^{\infty} \int_{\mathbb{R}^{d}} u(t, x) \delta W(t, x)=W(\mathbf{E}[u])+\sum_{n=1}^{\infty} I_{n+1}\left(\widetilde{f}_{n}\right),
$$

where $\tilde{f}_{n}$ denotes the symmetrization of $f_{n}$ in all its $n+1$ variables. We note here that if $\Lambda_{H}$ denotes the space of predictable processes $g$ defined on $\mathbb{R}_{+} \times \mathbb{R}$ such that almost surely $g \in \mathfrak{H}$ and $\mathbf{E}\left[\|g\|_{\mathfrak{H}}^{2}\right]<\infty$, the Skorohod integral of $g$ with respect to $W$ coincides with the Itô integral defined in [9], also, we have the isometry

$$
\mathbf{E}\left[\left(\int_{\mathbb{R}_{+}} \int_{\mathbb{R}} g(s, x) W(d s, d x)\right)^{2}\right]=\mathbb{E}\|g\|_{\mathfrak{H}}^{2} .
$$

Now we are ready to state the definition of the solution to equation (1.2).

Definition 2.2. Let $u=\{u(t, x), 0 \leq t \leq T, x \in \mathbb{R}\}$ be a real-valued predictable stochastic process such that for all $t \in[0, T]$ and $x \in \mathbb{R}$ the process $\left\{p_{t-s}(x-y) u(s, y) \mathbf{1}_{[0, t]}(s), 0 \leq s \leq\right.$ $t, y \in \mathbb{R}\}$ is Skorohod integrable, where $p_{t}(x)$ is the heat kernel on the real line related to $\frac{\kappa}{2} \Delta$. We say that $u$ is a mild solution of (1.2) if for all $t \in[0, T]$ and $x \in \mathbb{R}$ we have

$$
u(t, x)=p_{t} * u_{0}(x)+\int_{0}^{t} \int_{\mathbb{R}} p_{t-s}(x-y) u(s, y) W(d s, d y) \quad \text { a.s. },
$$

where the stochastic integral is understood in the sense of Skorohod or Itô.

\section{Existence and uniqueness}

In this section we prove the existence and uniqueness result for the solution to equation (1.2) by means of two different methods: one is via Fourier transform and the other is via chaos expansion. 


\subsection{Existence and uniqueness via Fourier transform}

In this subsection we discuss the existence and uniqueness of equation (1.2) using techniques of Fourier analysis.

Let $\dot{H}_{0}^{\frac{1}{2}-H}$ be the set of functions $f \in L^{2}(\mathbb{R})$ such that $\int_{\mathbb{R}}|\mathcal{F} f(\xi)|^{2}|\xi|^{1-2 H} d \xi<\infty$. This spaces is the time independent analogue to the space $\mathfrak{H}_{0}$ introduced in Proposition 2.1. We know that $\dot{H}_{0}^{\frac{1}{2}-H}$ is not complete with the seminorm $\left[\int_{\mathbb{R}}|\mathcal{F} f(\xi)|^{2}|\xi|^{1-2 H} d \xi\right]^{\frac{1}{2}}$ (see [17]). However, it is not difficult to check that the space $\dot{H}_{0}^{\frac{1}{2}-H}$ is complete for the seminorm $\|f\|_{\mathcal{V}(H)}^{2}:=\int_{\mathbb{R}}|\mathcal{F} f(\xi)|^{2}\left(1+|\xi|^{1-2 H}\right) d \xi$

In the next theorem we show the existence and uniqueness result assuming that the initial condition belongs to $\dot{H}_{0}^{\frac{1}{2}-H}$ and using estimates based on the Fourier transform in the space variable. To this purpose, we introduce the space $\mathcal{V}_{T}(H)$ as the completion of the set of elementary $\dot{H}_{0}^{\frac{1}{2}-H}$-valued stochastic processes $\{u(t, \cdot), t \in[0, T]\}$ with respect to the seminorm

$$
\|u\|_{\mathcal{V}_{T}(H)}^{2}:=\sup _{t \in[0, T]} \mathbf{E}\|u(t, \cdot)\|_{\mathcal{V}(H)}^{2}
$$

We now state a convolution lemma.

Proposition 3.1. Consider a function $u_{0} \in \dot{H}_{0}^{\frac{1}{2}-H}$ and $\frac{1}{4}<H<\frac{1}{2}$. For any $v \in \mathcal{V}_{T}(H)$ we set $\Gamma(v)=V$ in the following way:

$$
\Gamma(v):=V(t, x)=p_{t} * u_{0}(x)+\int_{0}^{t} \int_{\mathbb{R}} p_{t-s}(x-y) v(s, y) W(d s, d y), \quad t \in[0, T], x \in \mathbb{R} .
$$

Then $\Gamma$ is well-defined as a map from $\mathcal{V}_{T}(H)$ to $\mathcal{V}_{T}(H)$. Furthermore, there exist two positive constants $c_{1}, c_{2}$ such that the following estimate holds true on $[0, T]$ :

$$
\|V(t, \cdot)\|_{\mathcal{V}(H)}^{2} \leq c_{1}\left\|u_{0}\right\|_{\mathcal{V}(H)}^{2}+c_{2} \int_{0}^{t}(t-s)^{2 H-3 / 2}\|v(s, \cdot)\|_{\mathcal{V}(H)}^{2} d s .
$$

Proof. Let $v$ be a process in $\mathcal{V}_{T}(H)$ and set $V=\Gamma(v)$. We focus on the bound (3.2) for $V$.

Notice that the Fourier transform of $V$ can be computed easily. Indeed, setting $v_{0}(t, x)=$ $p_{t} * u_{0}(x)$ and invoking a stochastic version of Fubini's theorem, we get

$$
\mathcal{F} V(t, \xi)=\mathcal{F} v_{0}(t, \xi)+\int_{0}^{t} \int_{\mathbb{R}}\left(\int_{\mathbb{R}} e^{i x \xi} p_{t-s}(x-y) d x\right) v(s, y) W(d s, d y) .
$$

According to the expression of $\mathcal{F} p_{t}$, we obtain

$$
\mathcal{F} V(t, \xi)=\mathcal{F} v_{0}(t, \xi)+\int_{0}^{t} \int_{\mathbb{R}} e^{-i \xi y} e^{-\frac{\kappa}{2}(t-s) \xi^{2}} v(s, y) W(d s, d y) .
$$

We now evaluate the quantity $\mathbf{E}\left[\int_{\mathbb{R}}|\mathcal{F} V(t, \xi)|^{2}|\xi|^{1-2 H} d \xi\right]$ in the definition of $\|V\|_{\mathcal{V}_{T}(H)}$ given by (3.1). We thus write

$$
\begin{aligned}
\mathbf{E}\left[\int_{\mathbb{R}}|\mathcal{F} V(t, \xi)|^{2}|\xi|^{1-2 H} d \xi\right] \leq 2 \int_{\mathbb{R}}\left|\mathcal{F} v_{0}(t, \xi)\right|^{2}|\xi|^{1-2 H} d \xi \\
\quad+2 \int_{\mathbb{R}} \mathbf{E}\left[\left|\int_{0}^{t} \int_{\mathbb{R}} e^{-i \xi y} e^{-\frac{\kappa}{2}(t-s) \xi^{2}} v(s, y) W(d s, d y)\right|^{2}\right]|\xi|^{1-2 H} d \xi:=2\left(I_{1}+I_{2}\right),
\end{aligned}
$$


and we handle the terms $I_{1}$ and $I_{2}$ separately.

The term $I_{1}$ can be easily bounded by using that $u_{0} \in \dot{H}_{0}^{\frac{1}{2}-H}$ and recalling $v_{0}=p_{t} * u_{0}$. That is,

$$
I_{1}=\int_{\mathbb{R}}\left|\mathcal{F} u_{0}(\xi)\right|^{2} e^{-\kappa t|\xi|^{2}}|\xi|^{1-2 H} d \xi \leq C\left\|u_{0}\right\|_{\mathcal{V}(H)}^{2}
$$

We thus focus on the estimation of $I_{2}$, and we set $f_{\xi}(s, \eta)=e^{-i \xi \eta} e^{-\frac{\kappa}{2}(t-s) \xi^{2}} v(s, \eta)$. Applying the isometry property (2.8) we have:

$$
\mathbf{E}\left[\left|\int_{0}^{t} \int_{\mathbb{R}} e^{-i \xi y} e^{-\frac{\kappa}{2}(t-s) \xi^{2}} v(s, y) W(d s, d y)\right|^{2}\right]=c_{1, H} \int_{0}^{t} \int_{\mathbb{R}} \mathbf{E}\left[\left|\mathcal{F}_{\eta} f_{\xi}(s, \eta)\right|^{2}\right]|\eta|^{1-2 H} d s d \eta,
$$

where $\mathcal{F}_{\eta}$ is the Fourier transform with respect to $\eta$. It is obvious that the Fourier transform of $e^{-i \xi y} V(y)$ is $\mathcal{F} V(\eta+\xi)$. Thus we have

$$
\begin{aligned}
I_{2} & =C \int_{0}^{t} \int_{\mathbb{R}} \int_{\mathbb{R}} e^{-\kappa(t-s) \xi^{2}} \mathbf{E}\left[|\mathcal{F} v(s, \eta+\xi)|^{2}\right]|\eta|^{1-2 H}|\xi|^{1-2 H} d \eta d \xi d s \\
& =C \int_{0}^{t} \int_{\mathbb{R}} \int_{\mathbb{R}} e^{-\kappa(t-s) \xi^{2}} \mathbf{E}\left[|\mathcal{F} v(s, \eta)|^{2}\right]|\eta-\xi|^{1-2 H}|\xi|^{1-2 H} d \eta d \xi d s
\end{aligned}
$$

We now bound $|\eta-\xi|^{1-2 H}$ by $|\eta|^{1-2 H}+|\xi|^{1-2 H}$, which yields $I_{2} \leq I_{21}+I_{22}$ with:

$$
\begin{aligned}
& I_{21}=C \int_{0}^{t} \int_{\mathbb{R}} \int_{\mathbb{R}} e^{-\kappa(t-s) \xi^{2}} \mathbf{E}\left[|\mathcal{F} v(s, \eta)|^{2}\right]|\eta|^{1-2 H}|\xi|^{1-2 H} d \eta d \xi d s \\
& I_{22}=C \int_{0}^{t} \int_{\mathbb{R}} \int_{\mathbb{R}} e^{-\kappa(t-s) \xi^{2}} \mathbf{E}\left[|\mathcal{F} v(s, \eta)|^{2}\right]|\xi|^{2-4 H} d \eta d \xi d s
\end{aligned}
$$

Performing the change of variable $\xi \rightarrow(t-s)^{1 / 2} \xi$ and then trivially bounding the integrals of the form $\int_{\mathbb{R}}|\xi|^{\beta} e^{-\kappa \xi^{2}} d \xi$ by constants, we end up with

$$
\begin{aligned}
& I_{21} \leq C \int_{0}^{t}(t-s)^{H-1} \int_{\mathbb{R}} \mathbf{E}\left[|\mathcal{F} v(s, \eta)|^{2}\right]|\eta|^{1-2 H} d \eta d s \\
& I_{22} \leq C \int_{0}^{t}(t-s)^{2 H-3 / 2} \int_{\mathbb{R}} \mathbf{E}\left[|\mathcal{F} v(s, \eta)|^{2}\right] d \eta d s
\end{aligned}
$$

Observe that for $H \in\left(\frac{1}{4}, \frac{1}{2}\right)$ the term $(t-s)^{2 H-3 / 2}$ is more singular than $(t-s)^{H-1}$, but we still have $2 H-\frac{3}{2}>-1$ (this is where we need to impose $H>1 / 4$ ). Summarizing our consideration up to now, we have thus obtained

$$
\begin{aligned}
\int_{\mathbb{R}} \mathbf{E}[ & {\left[\left.\mathcal{F} V(t, \xi)\right|^{2}\right]|\xi|^{1-2 H} d \xi } \\
& \leq C_{1, T}\left\|u_{0}\right\|_{\mathcal{V}(H)}^{2}+C_{2, T} \int_{0}^{t}(t-s)^{2 H-3 / 2} \int_{\mathbb{R}} \mathbf{E}\left[|\mathcal{F} v(s, \xi)|^{2}\right]\left(1+|\xi|^{1-2 H}\right) d \xi d s
\end{aligned}
$$

for two strictly positive constants $C_{1, T}, C_{2, T}$.

The term $\mathbf{E}\left[\int_{\mathbb{R}}|\mathcal{F} V(t, \xi)|^{2} d \xi\right]$ in the definition of $\|V\|_{\mathcal{V}_{T}(H)}$ can be bounded with the same computations as above, and we find 


$$
\begin{aligned}
\int_{\mathbb{R}} \mathbf{E}\left[|\mathcal{F} V(t, \xi)|^{2}\right] d \xi & \\
& \leq C_{1, T}\left\|u_{0}\right\|_{\mathcal{V}(H)}^{2}+C_{2, T} \int_{0}^{t}(t-s)^{H-1} \int_{\mathbb{R}} \mathbf{E}\left[|\mathcal{F} v(s, \xi)|^{2}\right]\left(1+|\xi|^{1-2 H}\right) d \eta d s
\end{aligned}
$$

Hence, gathering our estimates (3.3) and (3.4), our bound (3.2) is easily obtained, which finishes the proof.

As in the forthcoming general case, Proposition 3.1 is the key to the existence and uniqueness result for equation (1.2).

Theorem 3.2. Suppose that $u_{0}$ is an element of $\dot{H}_{0}^{\frac{1}{2}-H}$ and $\frac{1}{4}<H<\frac{1}{2}$. Fix $T>0$. Then there is a unique process $u$ in the space $\mathcal{V}_{T}(H)$ such that for all $t \in[0, T]$,

$$
u(t, \cdot)=p_{t} * u_{0}+\int_{0}^{t} \int_{\mathbb{R}} p_{t-s}(\cdot-y) u(s, y) W(d s, d y) .
$$

Proof. The proof follows from the standard Picard iteration scheme, where we just set $u_{n+1}=$ $\Gamma\left(u_{n}\right)$. Details are left to the reader for sake of conciseness.

\subsection{Existence and uniqueness via chaos expansions}

Next, we provide another way to prove the existence and uniqueness of the solution to equation (1.2), by means of chaos expansions. This will enable us to obtain moment estimates. Before stating our main theorem in this direction, let us label an elementary lemma borrowed from [10] for further use.

Lemma 3.3. For $m \geq 1$ let $\alpha \in(-1+\varepsilon, 1)^{m}$ with $\varepsilon>0$ and set $|\alpha|=\sum_{i=1}^{m} \alpha_{i}$. For $t \in[0, T]$, the $m$-th dimensional simplex over $[0, t]$ is denoted by $T_{m}(t)=\left\{\left(r_{1}, r_{2}, \ldots, r_{m}\right) \in\right.$ $\left.\mathbb{R}^{m}: 0<r_{1}<\cdots<r_{m}<t\right\}$. Then there is a constant $c>0$ such that

$$
J_{m}(t, \alpha):=\int_{T_{m}(t)} \prod_{i=1}^{m}\left(r_{i}-r_{i-1}\right)^{\alpha_{i}} d r \leq \frac{c^{m} t^{|\alpha|+m}}{\Gamma(|\alpha|+m+1)},
$$

where by convention, $r_{0}=0$.

Let us now state a new existence and uniqueness theorem for our equation of interest.

Theorem 3.4. Suppose that $\frac{1}{4}<H<\frac{1}{2}$ and that the initial condition $u_{0}$ satisfies

$$
\int_{\mathbb{R}}\left(1+|\xi|^{\frac{1}{2}-H}\right)\left|\mathcal{F} u_{0}(\xi)\right| d \xi<\infty .
$$

Then there exists a unique solution to equation (1.2), that is, there is a unique process $u$ such that $p_{t-.}(x-\cdot) u$ is Skorohod integrable for any $(t, x) \in[0, T] \times \mathbb{R}$ and relation (2.9) holds true.

Remark 3.5. (i) The formulation of Theorem 3.4 yields the definition of our solution $u$ for all $(t, x) \in[0, T] \times \mathbb{R}$. This is in contrast with Theorem 3.2 which gives a solution sitting in $\dot{H}_{0}^{\frac{1}{2}-H}$ for every value of $t$, and thus defined a.e. in $x$ only. (ii) Condition (3.6) is satisfied by constant functions. 
Remark 3.6. In the later paper [12], the existence and uniqueness in Theorem 3.4 is obtained under a more general initial condition. Since the proof of Theorem 3.4 for condition (3.6) is easier and shorter, we present the proof as follows.

Proof of Theorem 3.4. Suppose that $u=\left\{u(t, x), t \geq 0, x \in \mathbb{R}^{d}\right\}$ is a solution to equation (2.9) in $\Lambda_{H}$. Then according to $(2.5)$, for any fixed $(t, x)$ the random variable $u(t, x)$ admits the following Wiener chaos expansion

$$
u(t, x)=\sum_{n=0}^{\infty} I_{n}\left(f_{n}(\cdot, t, x)\right)
$$

where for each $(t, x), f_{n}(\cdot, t, x)$ is a symmetric element in $\mathfrak{H}^{\otimes n}$. Hence, thanks to (2.7) and using an iteration procedure, one can find an explicit formula for the kernels $f_{n}$ for $n \geq 1$. Indeed, we have:

$$
\begin{aligned}
& f_{n}\left(s_{1}, x_{1}, \ldots, s_{n}, x_{n}, t, x\right) \\
& =\frac{1}{n !} p_{t-s_{\sigma(n)}}\left(x-x_{\sigma(n)}\right) \cdots p_{s_{\sigma(2)}-s_{\sigma(1)}}\left(x_{\sigma(2)}-x_{\sigma(1)}\right) p_{s_{\sigma(1)}} u_{0}\left(x_{\sigma(1)}\right),
\end{aligned}
$$

where $\sigma$ denotes the permutation of $\{1,2, \ldots, n\}$ such that $0<s_{\sigma(1)}<\cdots<s_{\sigma(n)}<t$ (see, for instance, formula (4.4) in [8] or formula (3.3) in [10]). Then, to show the existence and uniqueness of the solution it suffices to prove that for all $(t, x)$ we have

$$
\sum_{n=0}^{\infty} n !\left\|f_{n}(\cdot, t, x)\right\|_{\mathfrak{H}^{\otimes n}}^{2}<\infty
$$

The remainder of the proof is devoted to prove relation (3.9).

Starting from relation (3.8), some elementary Fourier computations show that

$$
\begin{aligned}
\mathcal{F} f_{n}\left(s_{1}, \xi_{1}, \ldots, s_{n}, \xi_{n}, t, x\right)= & \frac{c_{H}^{n}}{n !} \int_{\mathbb{R}} \prod_{i=1}^{n} e^{-\frac{\kappa}{2}\left(s_{\sigma(i+1)}-s_{\sigma(i)}\right)\left|\xi_{\sigma(i)}+\cdots+\xi_{\sigma(1)}-\zeta\right|^{2}} \\
& \times e^{-i x\left(\xi_{\sigma(n)}+\cdots+\xi_{\sigma(1)}-\zeta\right)} \mathcal{F} u_{0}(\zeta) e^{-\frac{\kappa s_{\sigma(1)}|\zeta|^{2}}{2}} d \zeta,
\end{aligned}
$$

where we have set $s_{\sigma(n+1)}=t$. Hence, owing to formula (2.3) for the norm in $\mathfrak{H}$ (in its Fourier mode version), we have

$$
\begin{array}{r}
n !\left\|f_{n}(\cdot, t, x)\right\|_{\mathfrak{H}^{\otimes n}}^{2}=\frac{c_{H}^{2 n}}{n !} \int_{[0, t]^{n}} \int_{\mathbb{R}^{n}} \mid \int_{\mathbb{R}} \prod_{i=1}^{n} e^{-\frac{\kappa}{2}\left(s_{\sigma(i+1)}-s_{\sigma(i)}\right)\left|\xi_{i}+\cdots+\xi_{1}-\zeta\right|^{2}} e^{-i x\left(\xi_{\sigma(n)}+\cdots+\xi_{\sigma(1)}-\zeta\right)} \\
\left.\mathcal{F} u_{0}(\zeta) e^{-\frac{\kappa s_{\sigma(1)}|\zeta|^{2}}{2}} d \zeta\right|^{2} \times \prod_{i=1}^{n}\left|\xi_{i}\right|^{1-2 H} d \xi d s, \quad(3 .)
\end{array}
$$

where $d \xi$ denotes $d \xi_{1} \cdots d \xi_{n}$ and similarly for $d s$. Then using the change of variable $\xi_{i}+$ $\cdots+\xi_{1}=\eta_{i}$, for all $i=1,2, \ldots, n$ and a linearization of the above expression, we obtain

$$
n !\left\|f_{n}(\cdot, t, x)\right\|_{\mathfrak{H}^{\otimes n}}^{2}=\frac{c_{H}^{2 n}}{n !} \int_{[0, t]^{n}} \int_{\mathbb{R}^{n}} \int_{\mathbb{R}^{2}} \prod_{i=1}^{n} e^{-\frac{\kappa}{2}\left(s_{\sigma(i+1)}-s_{\sigma(i)}\right)\left(\left|\eta_{i}-\zeta\right|^{2}+\left|\eta_{i}-\zeta^{\prime}\right|^{2}\right)} \mathcal{F} u_{0}(\zeta) \overline{\mathcal{F} u_{0}\left(\zeta^{\prime}\right)}
$$




$$
\times e^{i x\left(\zeta-\zeta^{\prime}\right)} e^{-\frac{\kappa s_{\sigma(1)}\left(|\zeta|^{2}+\left|\zeta^{\prime}\right|^{2}\right)}{2}} \prod_{i=1}^{n}\left|\eta_{i}-\eta_{i-1}\right|^{1-2 H} d \zeta d \zeta^{\prime} d \eta d s
$$

where we have set $\eta_{0}=0$. Then we use Cauchy-Schwarz inequality and bound the term $\exp \left(-\kappa s_{\sigma(1)}\left(|\zeta|^{2}+\left|\zeta^{\prime}\right|^{2}\right) / 2\right)$ by 1 to get

$$
\begin{aligned}
& n !\left\|f_{n}(\cdot, t, x)\right\|_{\mathfrak{H}^{\otimes n}}^{2} \leq \frac{c_{H}^{2 n}}{n !} \int_{\mathbb{R}^{2}}\left(\int_{[0, t]^{n}} \int_{\mathbb{R}^{n}} \prod_{i=1}^{n} e^{-\kappa\left(s_{\sigma(i+1)}-s_{\sigma(i)}\right)\left|\eta_{i}-\zeta\right|^{2}} \prod_{i=1}^{n}\left|\eta_{i}-\eta_{i-1}\right|^{1-2 H} d \eta d s\right)^{\frac{1}{2}} \\
& \times\left(\int_{[0, t]^{n}} \int_{\mathbb{R}^{n}} \prod_{i=1}^{n} e^{-\kappa\left(s_{\sigma(i+1)}-s_{\sigma(i)}\right)\left|\eta_{i}-\zeta^{\prime}\right|^{2}} \prod_{i=1}^{n}\left|\eta_{i}-\eta_{i-1}\right|^{1-2 H} d \eta d s\right)^{\frac{1}{2}}\left|\mathcal{F} u_{0}(\zeta)\right|\left|\mathcal{F} u_{0}\left(\zeta^{\prime}\right)\right| d \zeta d \zeta^{\prime} .
\end{aligned}
$$

Arranging the integrals again, performing the change of variables $\eta_{i}:=\eta_{i}-\zeta$ and invoking the trivial bound $\left|\eta_{i}-\eta_{i-1}\right|^{1-2 H} \leq\left|\eta_{i-1}\right|^{1-2 H}+\left|\eta_{i}\right|^{1-2 H}$, this yields

$$
n !\left\|f_{n}(\cdot, t, x)\right\|_{\mathfrak{H}^{\otimes n}}^{2} \leq \frac{c_{H}^{2 n}}{n !}\left(\int_{\mathbb{R}} L_{n, t}^{\frac{1}{2}}(\zeta)\left|\mathcal{F} u_{0}(\zeta)\right| d \zeta\right)^{2}
$$

where $L_{n, t}(\zeta)$ is

$$
\int_{[0, t]^{n}} \int_{\mathbb{R}^{n}} \prod_{i=1}^{n} e^{-\kappa\left(s_{\sigma(i+1)}-s_{\sigma(i)}\right)\left|\eta_{i}\right|^{2}}\left(|\zeta|^{1-2 H}+\left|\eta_{1}\right|^{1-2 H}\right) \times \prod_{i=2}^{n}\left(\left|\eta_{i}\right|^{1-2 H}+\left|\eta_{i-1}\right|^{1-2 H}\right) d \eta d s .
$$

Let us expand the product $\prod_{i=2}^{n}\left(\left|\eta_{i}\right|^{1-2 H}+\left|\eta_{i-1}\right|^{1-2 H}\right)$ in the integral defining $L_{n, t}(\zeta)$. We obtain an expression of the form $\sum_{\alpha \in D_{n}} \prod_{i=1}^{n}\left|\eta_{i}\right|^{\alpha_{i}}$, where $D_{n}$ is a subset of multi-indices of length $n-1$. The complete description of $D_{n}$ is omitted for sake of conciseness, and we will just use the following facts: $\operatorname{Card}\left(D_{n}\right)=2^{n-1}$ and for any $\alpha \in D_{n}$ we have

$$
|\alpha| \equiv \sum_{i=1}^{n} \alpha_{i}=(n-1)(1-2 H), \quad \text { and } \quad \alpha_{i} \in\{0,1-2 H, 2(1-2 H)\}, \quad i=1, \ldots, n .
$$

This simple expansion yields the following bound

$$
\begin{aligned}
L_{n, t}(\zeta) \leq|\zeta|^{1-2 H} \sum_{\alpha \in D_{n}} \int_{[0, t]^{n}} & \int_{\mathbb{R}^{n}} \prod_{i=1}^{n} e^{-\kappa\left(s_{\sigma(i+1)}-s_{\sigma(i)}\right)\left|\eta_{i}\right|^{2}} \prod_{i=1}^{n}\left|\eta_{i}\right|^{\alpha_{i}} d \eta d s \\
& \quad+\sum_{\alpha \in D_{n}} \int_{[0, t]^{n}} \int_{\mathbb{R}^{n}} \prod_{i=1}^{n} e^{-\kappa\left(s_{\sigma(i+1)}-s_{\sigma(i)}\right)\left|\eta_{i}\right|^{2}}\left|\eta_{1}\right|^{1-2 H} \prod_{i=1}^{n}\left|\eta_{i}\right|^{\alpha_{i}} d \eta d s .
\end{aligned}
$$

Perform the change of variable $\xi_{i}=\left(\kappa\left(s_{\sigma(i+1)}-s_{\sigma(i)}\right)\right)^{1 / 2} \eta_{i}$ in the above integral, and notice that $\int_{\mathbb{R}} e^{-\xi^{2}}|\xi|^{\alpha_{i}} d \xi$ is bounded by a constant for $\alpha_{i}>-1$. Changing the integral over $[0, t]^{n}$ into an integral over the simplex, we get

$$
\begin{aligned}
L_{n, t}(\zeta) \leq & C|\zeta|^{1-2 H} n ! c_{H}^{n} \sum_{\alpha \in D_{n}} \int_{T_{n}(t)} \prod_{i=1}^{n}\left(\kappa\left(s_{i+1}-s_{i}\right)\right)^{-\frac{1}{2}\left(1+\alpha_{i}\right)} d s \\
& +C n ! c_{H}^{n} \sum_{\alpha \in D_{n}} \int_{T_{n}(t)}\left(\kappa\left(s_{2}-s_{1}\right)\right)^{-\frac{2-2 H+\alpha_{1}}{2}} \prod_{i=2}^{n}\left(\kappa\left(s_{i+1}-s_{i}\right)\right)^{-\frac{1}{2}\left(1+\alpha_{i}\right)} d s
\end{aligned}
$$


We observe that whenever $\frac{1}{4}<H<\frac{1}{2}$, we have $\frac{1}{2}\left(1+\alpha_{i}\right)<1$ for all $i=2, \ldots n$, and it is easy to see that $\alpha_{1}$ is at most $1-2 H$ so $\frac{1}{2}\left(2-2 H+\alpha_{1}\right)<1$. (The condition $H>1 / 4$ comes from the requirement that when $\alpha_{1}=1-2 H$, we need $\frac{1}{2}\left(2-2 H+\alpha_{1}\right)=\frac{1}{2}(3-4 H)<1$. $)$ Thanks to Lemma 3.3 and recalling that $\sum_{i=1}^{n} \alpha_{i}=(n-1)(1-2 H)$ for all $\alpha \in D_{n}$, we thus conclude that

$$
L_{n, t}(\zeta) \leq \frac{C\left(1+t^{\frac{1}{2}-H} \kappa^{\frac{1}{2}-H}|\zeta|^{1-2 H}\right) n ! c^{n} c_{H}^{n} t^{n H} \kappa^{n H-n}}{\Gamma(n H+1)}
$$

Plugging this expression into (3.11), we end up with

$$
n !\left\|f_{n}(\cdot, t, x)\right\|_{\mathfrak{H}^{\otimes n}}^{2} \leq \frac{C c_{H}^{n} c^{n} t^{n H} \kappa^{n H-n}}{\Gamma(n H+1)}\left(\int_{\mathbb{R}}\left(1+t^{\frac{1}{2}-H} \kappa^{\frac{1}{2}-H}|\zeta|^{\frac{1}{2}-H}\right)\left|\mathcal{F} u_{0}(\zeta)\right| d \zeta\right)^{2} .
$$

The proof of (3.9) is now easily completed thanks to the asymptotic behavior of the Gamma function and our assumption of $u_{0}$, and this finishes the existence and uniqueness proof.

\section{Moment formula and bounds}

In this section we derive the Feynman-Kac formula for the moments of the solution to equation (1.2) and the upper and lower bounds for the moments of the solution to equation (1.2) which allow us to conclude on the intermittency of the solution. We proceed by first getting an approximation result for $u$, and then deriving the upper and lower bounds for the approximation.

\subsection{Approximation of the solution}

The approximation of the solution we consider is based on the following approximation of the noise $W$. For each $\varepsilon>0$ and $\varphi \in \mathfrak{H}$, we define

$$
W_{\varepsilon}(\varphi)=\int_{0}^{t} \int_{\mathbb{R}}\left[\rho_{\varepsilon} * \varphi\right](s, x) W(d s, d y)=\int_{0}^{t} \int_{\mathbb{R}} \int_{\mathbb{R}} \varphi(s, x) \rho_{\varepsilon}(x-y) W(d s, d y) d x,
$$

where $\rho_{\varepsilon}(x)=(2 \pi \varepsilon)^{-\frac{1}{2}} e^{-x^{2} /(2 \varepsilon)}$. Notice that the covariance of $W_{\varepsilon}$ can be read (either in Fourier or direct coordinates) as:

$$
\begin{aligned}
\mathbf{E}\left[W_{\varepsilon}(\varphi) W_{\varepsilon}(\psi)\right] & =c_{1, H} \int_{0}^{t} \int_{\mathbb{R}} \mathcal{F} \varphi(s, \xi) \overline{\mathcal{F} \psi(s, \xi)} e^{-\varepsilon|\xi|^{2}}|\xi|^{1-2 H} d \xi d s \\
& =c_{1, H} \int_{0}^{t} \int_{\mathbb{R}} \int_{\mathbb{R}} \varphi(s, x) f_{\varepsilon}(x-y) \psi(s, y) d x d y d s
\end{aligned}
$$

for every $\varphi, \psi$ in $\mathfrak{H}$, where $f_{\varepsilon}$ is given by $f_{\varepsilon}(x)=\mathcal{F}^{-1}\left(e^{-\varepsilon|\xi|^{2}}|\xi|^{1-2 H}\right)$. In other words, $W_{\varepsilon}$ is still a white noise in time but its space covariance is now given by $f_{\varepsilon}$. Note that $f_{\varepsilon}$ is a real positive-definite function, but is not necessarily positive. The noise $W_{\varepsilon}$ induces an approximation to the mild formulation of equation (1.2), namely

$$
u_{\varepsilon}(t, x)=p_{t} * u_{0}(x)+\int_{0}^{t} \int_{\mathbb{R}} p_{t-s}(x-y) u_{\varepsilon}(s, y) W_{\varepsilon}(d s, d y),
$$

where the integral is understood (as in Subsection 3.1) in the Itô sense. We will start by a formula for the moments of $u_{\varepsilon}$. 
Proposition 4.1. Let $W_{\varepsilon}$ be the noise defined by (4.1), and assume $\frac{1}{4}<H<\frac{1}{2}$. Assume $u_{0}$ is such that $\int_{\mathbb{R}}\left(1+|\xi|^{\frac{1}{2}-H}\right)\left|\mathcal{F} u_{0}(\xi)\right| d \xi<\infty$. Then

(i) Equation (4.3) admits a unique solution.

(ii) For any integer $n \geq 2$ and $(t, x) \in[0, T] \times \mathbb{R}$, we have

$$
\mathbf{E}\left[u_{\varepsilon}^{n}(t, x)\right]=\mathbf{E}_{B}\left[\prod_{j=1}^{n} u_{0}\left(x+B_{\kappa t}^{j}\right) \exp \left(c_{1, H} \sum_{1 \leq j \neq k \leq n} V_{t, x}^{\varepsilon, j, k}\right)\right],
$$

with

$$
V_{t, x}^{\varepsilon, j, k}=\int_{0}^{t} f_{\varepsilon}\left(B_{\kappa r}^{j}-B_{\kappa r}^{k}\right) d r=\int_{0}^{t} \int_{\mathbb{R}} e^{-\varepsilon|\xi|^{2}}|\xi|^{1-2 H} e^{i \xi\left(B_{\kappa r}^{j}-B_{\kappa r}^{k}\right)} d \xi d r .
$$

In formula (4.5), $\left\{B^{j} ; j=1, \ldots, n\right\}$ is a family of $n$ independent standard Brownian motions which are also independent of $W$ and $\mathbf{E}_{B}$ denotes the expected value with respect to the randomness in $B$ only.

(iii) The quantity $\mathbf{E}\left[u_{\varepsilon}^{n}(t, x)\right]$ is uniformly bounded in $\varepsilon$. More generally, for any $a>0$ we have

$$
\sup _{\varepsilon>0} \mathbf{E}_{B}\left[\exp \left(a \sum_{1 \leq j \neq k \leq n} V_{t, x}^{\varepsilon, j, k}\right)\right] \equiv c_{a}<\infty .
$$

Proof. The proof of item (i) is almost identical to the proof of Theorem 3.4, and is omitted for sake of conciseness. Moreover, in the proof of (ii) and (iii), we may take $u_{0}(x) \equiv 1$ for simplicity.

In order to check item (ii), set

$$
A_{t, x}^{\varepsilon}(r, y)=\rho_{\varepsilon}\left(B_{\kappa(t-r)}^{x}-y\right), \quad \text { and } \quad \alpha_{t, x}^{\varepsilon}=\left\|A_{t, x}^{\varepsilon}\right\|_{\mathfrak{H}}^{2} .
$$

Then one can prove, similarly to Proposition 5.2 in [8], that $u_{\varepsilon}$ admits a Feynman-Kac representation of the form

$$
u_{\varepsilon}(t, x)=\mathbf{E}_{B}\left[\exp \left(W\left(A_{t, x}^{\varepsilon}\right)-\frac{1}{2} \alpha_{t, x}^{\varepsilon}\right)\right] .
$$

Now fix an integer $n \geq 2$. According to (4.7) we have

$$
\mathbf{E}\left[u_{\varepsilon}^{n}(t, x)\right]=\mathbf{E}_{W}\left[\prod_{j=1}^{n} \mathbf{E}_{B}\left[\exp \left(W\left(A_{t, x}^{\varepsilon, B^{j}}\right)-\frac{1}{2} \alpha_{t, x}^{\varepsilon, B^{j}}\right)\right]\right],
$$

where for any $j=1, \ldots, n, A_{t, x}^{\varepsilon, B^{j}}$ and $\alpha_{t, x}^{\varepsilon, B^{j}}$ are evaluations of (4.6) using the Brownian motion $B^{j}$. Therefore, since $W\left(A_{t, x}^{\varepsilon, B^{j}}\right)$ is a Gaussian random variable conditionally on $B$, we obtain

$$
\begin{aligned}
\mathbf{E}\left[u_{\varepsilon}^{n}(t, x)\right] & =\mathbf{E}_{B}\left[\exp \left(\frac{1}{2}\left\|\sum_{j=1}^{n} A_{t, x}^{\varepsilon, B^{j}}\right\|_{\mathfrak{H}}^{2}-\frac{1}{2} \sum_{j=1}^{n} \alpha_{t, x}^{\varepsilon, B^{j}}\right)\right] \\
& =\mathbf{E}_{B}\left[\exp \left(\frac{1}{2}\left\|\sum_{j=1}^{n} A_{t, x}^{\varepsilon, B^{j}}\right\|_{\mathfrak{H}}^{2}-\frac{1}{2} \sum_{j=1}^{n}\left\|A_{t, x}^{\varepsilon, B^{j}}\right\|_{\mathfrak{H}}^{2}\right)\right]
\end{aligned}
$$




$$
=\mathbf{E}_{B}\left[\exp \left(\sum_{1 \leq i<j \leq n}\left\langle A_{t, x}^{\varepsilon, B^{i}}, A_{t, x}^{\varepsilon, B^{j}}\right\rangle_{\mathfrak{H}}\right)\right]
$$

The evaluation of $\left\langle A_{t, x}^{\varepsilon, B^{i}}, A_{t, x}^{\varepsilon, B^{j}}\right\rangle_{\mathfrak{H}}$ easily yields our claim (4.4), the last details being left to the patient reader.

Let us now prove item (iii), namely

$$
\sup _{\varepsilon>0} \sup _{t \in[0, T], x \in \mathbb{R}} \mathbf{E}\left[u_{\varepsilon}^{n}(t, x)\right]<\infty .
$$

To this aim, observe first that we have obtained an expression (4.4) which does not depend on $x \in \mathbb{R}$, so that the $\sup _{t \in[0, T], x \in \mathbb{R}}$ in (4.8) can be reduced to a sup in $t$ only. Next, still resorting to formula (4.4), it is readily seen that it suffices to show that for two independent Brownian motions $B$ and $\tilde{B}$, we have

$$
\sup _{\varepsilon>0, t \in[0, T]} \mathbf{E}_{B}\left[\exp \left(c F_{t}^{\varepsilon}\right)\right]<\infty, \quad \text { with } \quad F_{t}^{\varepsilon} \equiv \int_{0}^{t} \int_{\mathbb{R}} e^{-\varepsilon|\xi|^{2}}|\xi|^{1-2 H} e^{i \xi\left(B_{\kappa r}-\tilde{B}_{\kappa r}\right)} d \xi d r
$$

for any positive constant $c$. In order to prove (4.9), we expand the exponential and write:

$$
\mathbf{E}_{B}\left[\exp \left(c F_{t}^{\varepsilon}\right)\right]=\sum_{l=0}^{\infty} \frac{\mathbf{E}_{B}\left[\left(c F_{t}^{\varepsilon}\right)^{l}\right]}{l !}
$$

Next, we have

$$
\begin{aligned}
\mathbf{E}_{B}\left[\left(F_{t}^{\varepsilon}\right)^{l}\right] & =\mathbf{E}_{B}\left[\int_{[0, t]^{l}} \int_{\mathbb{R}^{l}} \prod_{j=1}^{l} e^{-i \xi_{j}\left(B_{\kappa r_{j}}-\tilde{B}_{\kappa r_{j}}\right)-\varepsilon\left|\xi_{j}\right|^{2}}\left|\xi_{j}\right|^{1-2 H} d \xi d r\right] \\
& \leq \int_{[0, t]^{l}} \int_{\mathbb{R}^{l}} \prod_{j=1}^{l} e^{-\kappa\left(t-r_{\sigma(j)}\right)\left|\xi_{j}+\cdots+\xi_{1}\right|^{2}}\left|\xi_{j}\right|^{1-2 H} d \xi d r,
\end{aligned}
$$

where $\sigma$ is the permutation on $\{1,2, \ldots, l\}$ such that $t \geq r_{\sigma(l)} \geq \cdots \geq r_{\sigma(1)}$. We have thus gone back to an expression which is very similar to (3.10). We now proceed as in the proof of Theorem 3.4 to show that (4.8) holds true from equation (4.10).

Starting from Proposition 4.1, let us take limits in order to get the moment formula for the solution $u$ to equation (1.2).

Theorem 4.2. Assume $\frac{1}{4}<H<\frac{1}{2}$ and consider $n \geq 1, j, k \in\{1, \ldots, n\}$ with $j \neq k$. For $(t, x) \in[0, T] \times \mathbb{R}$, denote by $V_{t, x}^{j, k}$ the limit in $L^{2}(\Omega)$ as $\varepsilon \rightarrow 0$ of

$$
V_{t, x}^{\varepsilon, j, k}=\int_{0}^{t} \int_{\mathbb{R}} e^{-\varepsilon|\xi|^{2}}|\xi|^{1-2 H} e^{i \xi\left(B_{\kappa r}^{j}-B_{\kappa r}^{k}\right)} d \xi d r .
$$

Then $\mathbf{E}\left[u_{\varepsilon}^{n}(t, x)\right]$ converges as $\varepsilon \rightarrow 0$ to $\mathbf{E}\left[u^{n}(t, x)\right]$, which is given by

$$
\mathbf{E}\left[u^{n}(t, x)\right]=\mathbf{E}_{B}\left[\prod_{j=1}^{n} u_{0}\left(B_{\kappa t}^{j}+x\right) \exp \left(c_{1, H} \sum_{1 \leq j \neq k \leq n} V_{t, x}^{j, k}\right)\right] .
$$


We note that in a recent paper [12], the moment formula for general covariance function is obtained. However we present the proof here for the sake of completeness.

Proof. As in Proposition 4.1, we will prove the theorem for $u_{0} \equiv 1$ for simplicity. For any $p \geq 1$ and $1 \leq j<k \leq n$, we can easily prove that $V_{t, x}^{\varepsilon, j, k}$ converges in $L^{p}(\Omega)$ to $V_{t, x}^{j, k}$ defined by

$$
V_{t, x}^{j, k}=\int_{0}^{t} \int_{\mathbb{R}}|\xi|^{1-2 H} e^{i \xi\left(B_{\kappa r}^{j}-B_{\kappa r}^{k}\right)} d \xi d r
$$

Indeed, this is due to the fact that $e^{-\varepsilon|\xi|^{2}}|\xi|^{1-2 H} e^{i \xi\left(B_{\kappa r}^{j}-B_{\kappa r}^{k}\right)}$ converges to $|\xi|^{1-2 H} e^{i \xi\left(B_{\kappa r}^{j}-B_{\kappa r}^{k}\right)}$ in the $d \xi \otimes d r \otimes d \mathbf{P}$ sense, plus standard uniform integrability arguments. Now, taking into account relation (4.4), Proposition 4.1 (iii), the fact that $V_{t, x}^{\varepsilon, j, k}$ converges to $V_{t, x}^{j, k}$ in $L^{2}(\Omega)$ as $\varepsilon \rightarrow 0$, and the inequality $\left|e^{x}-e^{y}\right| \leq\left(e^{x}+e^{y}\right)|x-y|$, we obtain

$$
\begin{aligned}
& \mathbf{E}_{B}\left|\exp \left(c_{1, H} \sum_{1 \leq j \neq k \leq n} V_{t, x}^{\epsilon, j, k}\right)-\exp \left(c_{1, H} \sum_{1 \leq j \neq k \leq n} V_{t, x}^{j, k}\right)\right| \\
\leq & \sup _{\epsilon>0} 2\left(\mathbf{E}_{B}\left|\exp \left(2 c_{1, H} \sum_{1 \leq j \neq k \leq n} V_{t, x}^{\epsilon, j, k}\right)+\exp \left(2 c_{1, H} \sum_{1 \leq j \neq k \leq n} V_{t, x}^{j, k}\right)\right|^{2}\right)^{\frac{1}{2}} \\
\times & \left(\mathbf{E}_{B}\left|c_{1, H} \sum_{1 \leq j \neq k \leq n} V_{t, x}^{\epsilon, j, k}-c_{1, H} \sum_{1 \leq j \neq k \leq n} V_{t, x}^{j, k}\right|^{2}\right)^{\frac{1}{2}},
\end{aligned}
$$

which implies

$$
\begin{aligned}
\lim _{\varepsilon \rightarrow 0} \mathbf{E}\left[u_{\varepsilon}^{n}(t, x)\right] & =\lim _{\varepsilon \rightarrow 0} \mathbf{E}_{B}\left[\exp \left(c_{1, H} \sum_{1 \leq j \neq k \leq n} V_{t, x}^{\varepsilon, j, k}\right)\right] \\
& =\mathbf{E}_{B}\left[\exp \left(c_{1, H} \sum_{1 \leq j \neq k \leq n} V_{t, x}^{j, k}\right)\right] .
\end{aligned}
$$

To end the proof, let us now identify the right hand side of (4.13) with $\mathbf{E}\left[u^{n}(t, x)\right]$, where $u$ is the solution to equation (1.2). For $\varepsilon, \varepsilon^{\prime}>0$ we write

$$
\mathbf{E}\left[u_{\varepsilon}(t, x) u_{\varepsilon^{\prime}}(t, x)\right]=\mathbf{E}_{B}\left[\exp \left(\left\langle A_{t, x}^{\varepsilon, B^{1}}, A_{t, x}^{\varepsilon^{\prime}, B^{2}}\right\rangle_{\mathfrak{H}}\right)\right]
$$

where we recall that $A_{t, x}^{\varepsilon, B}$ is defined by relation (4.6). As before we can show that this converges as $\varepsilon, \varepsilon^{\prime}$ tend to zero. So, $u_{\varepsilon}(t, x)$ converges in $L^{2}$ to some limit $v(t, x)$, and the limit is actually in $L^{p}$, for all $p \geq 1$. Moreover, $\mathbf{E}\left[v^{k}(t, x)\right]$ is equal to the right hand side of (4.13). Finally, for any smooth random variable $F$ which is a linear combination of $W\left(\mathbf{1}_{[a, b]}(s) \varphi(x)\right)$, where $\varphi$ is a $C^{\infty}$ function with compact support, using the duality relation (2.4), we have

$$
\mathbf{E}\left[F u_{\varepsilon}(t, x)\right]=\mathbf{E}[F]+\mathbf{E}\left[\left\langle Y^{\varepsilon}, D F\right\rangle_{\mathfrak{H}}\right],
$$

where

$$
Y^{t, x}(s, z)=\left(\int_{\mathbb{R}} p_{t-s}(x-y) p_{\varepsilon}(y-z) u_{\varepsilon}(s, y) d y\right) \mathbf{1}_{[0, t]}(s)
$$


Letting $\varepsilon$ tend to zero in equation (4.14), after some easy calculation we get

$$
\mathbf{E}\left[F v_{t, x}\right]=\mathbf{E}[F]+\mathbf{E}\left[\left\langle D F, v p_{t-.}(x-\cdot)\right\rangle_{\mathfrak{H}}\right] .
$$

This equation is valid for any $F \in \mathbb{D}^{1,2}$ by approximation. So the above equation implies that the process $v$ is the solution of equation (1.2), and by the uniqueness of the solution we have $v=u$.

\subsection{Intermittency estimates}

In this subsection we prove some upper and lower bounds on the moments of the solution which entail the intermittency phenomenon.

Theorem 4.3. Let $\frac{1}{4}<H<\frac{1}{2}$, and consider the solution $u$ to equation (1.2). For simplicity we assume that the initial condition is $u_{0}(x) \equiv 1$. Let $n \geq 2$ be an integer, $x \in \mathbb{R}$ and $t \geq 0$. Then there exist some positive constants $c_{1}, c_{2}, c_{3}$ independent of $n, t$ and $\kappa$ with $0<c_{1}<c_{2}<\infty$ satisfying

$$
\exp \left(c_{1} n^{1+\frac{1}{H}} \kappa^{1-\frac{1}{H}} t\right) \leq \mathbf{E}\left[u^{n}(t, x)\right] \leq c_{3} \exp \left(c_{2} n^{1+\frac{1}{H}} \kappa^{1-\frac{1}{H}} t\right) .
$$

Proof of Theorem 4.3. We divide this proof into upper and lower bound estimates.

Step 1: Upper bound. Recall from equation (3.7) that for $(t, x) \in \mathbb{R}_{+} \times \mathbb{R}, u(t, x)$ can be written as: $u(t, x)=\sum_{m=0}^{\infty} I_{m}\left(f_{m}(\cdot, t, x)\right)$. Moreover, as a consequence of the hypercontractivity property on a fixed chaos we have (see $[16$, p. 62])

$$
\left\|I_{m}\left(f_{m}(\cdot, t, x)\right)\right\|_{L^{n}(\Omega)} \leq(n-1)^{\frac{m}{2}}\left\|I_{m}\left(f_{m}(\cdot, t, x)\right)\right\|_{L^{2}(\Omega)},
$$

and substituting the above right hand side by the bound (3.12), we end up with

$$
\left\|I_{m}\left(f_{m}(\cdot, t, x)\right)\right\|_{L^{n}(\Omega)} \leq n^{\frac{m}{2}}\left\|I_{m}\left(f_{m}(\cdot, t, x)\right)\right\|_{L^{2}(\Omega)} \leq \frac{c^{\frac{n}{2}} n^{\frac{m}{2}} t^{\frac{m H}{2}} \kappa^{\frac{H m-m}{2}}}{\Gamma(m H / 2+1)} .
$$

Therefore from by the asymptotic bound of Mittag-Leffler function $\sum_{n \geq 0} x^{n} / \Gamma(\alpha n+1) \leq$ $c_{1} \exp \left(c_{2} x^{1 / a}\right)$ (see [13], Formula (1.8.10)), we get:

$$
\|u(t, x)\|_{L^{n}(\Omega)} \leq \sum_{m=0}^{\infty}\left\|J_{m}(t, x)\right\|_{L^{n}(\Omega)} \leq \sum_{m=0}^{\infty} \frac{c^{\frac{m}{2}} n^{\frac{m}{2}} t^{\frac{m H}{2}} \kappa^{\frac{H m-m}{2}}}{(\Gamma(m H+1))^{\frac{1}{2}}} \leq c_{1} \exp \left(c_{2} t n^{\frac{1}{H}} \kappa^{\frac{H-1}{H}}\right),
$$

from which the upper bound in our theorem is easily deduced.

Step 2: Lower bound for $u_{\varepsilon}$. For the lower bound, we start from the moment formula (4.4) for the approximate solution, and write

$$
\begin{aligned}
& \mathbf{E}\left[u_{\varepsilon}^{n}(t, x)\right] \\
& =\mathbf{E}_{B}\left[\exp \left(c_{1, H}\left[\int_{0}^{t} \int_{\mathbb{R}} e^{-\varepsilon|\xi|^{2}}\left|\sum_{j=1}^{n} e^{-i B_{\kappa r}^{j} \xi}\right|^{2}|\xi|^{1-2 H} d \xi d r-n t \int_{\mathbb{R}} e^{-\varepsilon|\xi|^{2}}|\xi|^{1-2 H} d \xi\right]\right)\right] .
\end{aligned}
$$

In order to estimate the expression above, notice first that the obvious change of variable $\lambda=\varepsilon^{1 / 2} \xi$ yields $\int_{\mathbb{R}} e^{-\varepsilon|\xi|^{2}}|\xi|^{1-2 H} d \xi=C \varepsilon^{-(1-H)}$ for some constant $C$. Now for an additional arbitrary parameter $\eta>0$, consider the set

$$
A_{\eta}=\left\{\omega ; \sup _{1 \leq j \leq n} \sup _{0 \leq r \leq t}\left|B_{\kappa r}^{j}(\omega)\right| \leq \frac{\pi}{3 \eta}\right\} .
$$


Observe that classical small balls inequalities for a Brownian motion (see (1.3) in [15]) yield $\mathbf{P}\left(A_{\eta}\right) \geq c_{1} e^{-c_{2} \eta^{2} n \kappa t}$ for a large enough $\eta$. In addition, if we assume that $A_{\eta}$ is realized and $|\xi| \leq \eta$, some elementary trigonometric identities show that the following deterministic bound hold true: $\left|\sum_{j=1}^{n} e^{-i B_{\kappa r}^{j} \xi}\right| \geq \frac{n}{2}$. Gathering those considerations, we thus get

$$
\begin{aligned}
\mathbf{E}\left[u_{\varepsilon}^{n}(t, x)\right] & \geq \exp \left(c_{1} n^{2} \int_{0}^{t} \int_{0}^{\eta} e^{-\varepsilon|\xi|^{2}}|\xi|^{1-2 H} d \xi d r-c_{2} n t \varepsilon^{H-1}\right) \mathbf{P}\left(A_{\eta}\right) \\
& \geq C \exp \left(c_{1} n^{2} t \varepsilon^{-(1-H)} \int_{0}^{\varepsilon^{1 / 2} \eta} e^{-|\xi|^{2}}|\xi|^{1-2 H} d \xi-c_{2} n t \varepsilon^{-(1-H)}-c_{3} n \kappa t \eta^{2}\right) .
\end{aligned}
$$

We now choose the parameter $\eta$ such that $\kappa \eta^{2}=\varepsilon^{-(1-H)}$, which means in particular that $\eta \rightarrow \infty$ as $\varepsilon \rightarrow 0$. It is then easily seen that $\int_{0}^{\varepsilon^{1 / 2} \eta} e^{-|\xi|^{2}}|\xi|^{1-2 H} d \xi$ is of order $\varepsilon^{H(1-H)}$ in this regime, and some elementary algebraic manipulations entail

$$
\mathbf{E}\left[u_{\varepsilon}^{n}(t, x)\right] \geq C \exp \left(c_{1} n^{2} t \kappa^{H-1} \varepsilon^{-(1-H)^{2}}-c_{2} n t \varepsilon^{-(1-H)}\right) \geq C \exp \left(c_{3} t \kappa^{1-\frac{1}{H}} n^{1+\frac{1}{H}}\right),
$$

where the last inequality is obtained by choosing $\varepsilon^{-(1-H)}=c \kappa^{\frac{H-1}{H}} n^{\frac{1}{H}}$ in order to optimize the second expression. We have thus reached the desired lower bound in (4.15) for the approximation $u^{\varepsilon}$ in the regime $\varepsilon=c \kappa^{\frac{1}{H}} n^{-\frac{1}{H(1-H)}}$.

Step 3: Lower bound for $u$. To complete the proof, we need to show that for all sufficiently small $\varepsilon, \mathbf{E}\left[u_{\varepsilon}^{n}(t, x)\right] \leq \mathbf{E}\left[u^{n}(t, x)\right]$. We thus start from equation (4.4) and use the series expansion of the exponential function as in (4.10). We get

$$
\mathbf{E}\left[u_{\varepsilon}^{n}(t, x)\right]=\sum_{m=0}^{\infty} \frac{c_{1, H}^{m}}{m !} \mathbf{E}_{B}\left[\left(\sum_{1 \leq j \neq k \leq n} V_{t, x}^{\varepsilon, j, k}\right)^{m}\right]
$$

where we recall that $V_{t, x}^{\varepsilon, j, k}$ is defined by (4.5). Furthermore, expanding the $m$ th power above, we have

$$
\mathbf{E}_{B}\left[\left(\sum_{1 \leq j \neq k \leq n} V_{t, x}^{\varepsilon, j, k}\right)^{m}\right]=\sum_{\alpha \in K_{n, m}} \int_{[0, t]^{m}} \int_{\mathbb{R}^{m}} e^{-\varepsilon \sum_{l=1}^{m}\left|\xi_{l}\right|^{2}} \mathbf{E}_{B}\left[e^{i B^{\alpha}(\xi)}\right] \prod_{l=1}^{m}\left|\xi_{l}\right|^{1-2 H} d \xi d r,
$$

where $K_{n, m}$ is a set of multi-indices defined by

$$
K_{n, m}=\left\{\alpha=\left(j_{1}, \ldots, j_{m}, k_{1}, \ldots, k_{m}\right) \in\{1, \ldots, n\}^{2 m} ; j_{l}<k_{l} \text { for all } l=1, \ldots, n\right\},
$$

and $B^{\alpha}(\xi)$ is a shorthand for the linear combination $\sum_{l=1}^{m} \xi_{l}\left(B_{\kappa r_{l}}^{j_{l}}-B_{\kappa r_{l}}^{k_{l}}\right)$. The important point here is that $E_{B} e^{i B^{\alpha}(\xi)}$ is positive for any $\alpha \in K_{n, m}$. We thus get the following inequality, valid for all $m \geq 1$

$$
\begin{aligned}
\mathbf{E}_{B}\left[\left(\sum_{1 \leq j \neq k \leq n} V_{t, x}^{\varepsilon, j, k}\right)^{m}\right] & \leq \sum_{\alpha \in K_{n, m}} \int_{[0, t]^{m}} \int_{\mathbb{R}^{m}} \mathbf{E}_{B}\left[e^{i B^{\alpha}(\xi)}\right] \prod_{l=1}^{m}\left|\xi_{l}\right|^{1-2 H} d \xi d r \\
& =\mathbf{E}_{B}\left[\left(\sum_{1 \leq j \neq k \leq n} V_{t, x}^{j, k}\right)^{m}\right]
\end{aligned}
$$

where $V_{t, x}^{j, k}$ is defined by (4.12). Plugging this inequality back into (4.16) and recalling expression (4.11) for $\mathbf{E}\left[u^{n}(t, x)\right]$, we easily deduce that $\mathbf{E}\left[u_{\varepsilon}^{n}(t, x)\right] \leq \mathbf{E}\left[u^{n}(t, x)\right]$, which finishes the proof. 


\section{References}

[1] Alberts, T., Khanin, K., Quastel, J. The continuum directed random polymer. J. Stat. Phys. 154 (2014), no. 1-2, 305-326.

[2] Balan, R., Jolis, M. and Quer-Sardanyons, L. SPDEs with fractional noise in space with index $H<1 / 2$. Electron. J. Probab. Volume 20 (2015), paper no. 54, 36 pp.

[3] Bertini, L., Cancrini, N. The stochastic heat equation: Feynman- Kac formula and intermittence. J. Statist. Phys. 78 (1995), no. 5-6, 1377-1401.

[4] Bezerra, S., Tindel, S., Viens, F. Superdiffusivity for a Brownian polymer in a continuous Gaussian environment. Ann. Probab. 36 (2008), no. 5, 1642-1675.

[5] Chen, X. Spatial asymptotics for the parabolic Anderson models with generalized timespace Gaussian noise. Ann. Probab. Volume 44, Number 2 (2016), 1535-1598.

[6] Hairer, M. Solving the KPZ equation. Ann. of Math. (2) 178 (2013), no. 2, 559-664.

[7] Hu, Y. Analysis on Gaussian space. World Scientific, Singapore, 2017.

[8] Hu, Y., Nualart, D. Stochastic heat equation driven by fractional noise and local time. Probab. Theory Related Fields 143 (2009), no. 1-2, 285-328.

[9] Hu, Y., Huang, J., Lê, K., Nualart, D. and Tindel, S. Stochastic heat equation with rough dependence in space. Ann. Probab. pending revision.

[10] Hu, Y., Huang, J., Nualart, D., Tindel, S. Stochastic heat equations with general multiplicative Gaussian noises: Hölder continuity and intermittency. Electron. J. Probab. 20 (2015), no. 55, 50 pp.

[11] Hu, Y., Nualart, D., Song, J. Feynman-Kac formula for heat equation driven by fractional white noise. Ann. Probab. 30, 291-326.

[12] Huang, J., Lê, K., Nualart, D. Large time asymptotics for the parabolic Anderson model driven by spatially correlated noise. Ann. Inst. H. Poincaré, to appear.

[13] Kilbas,A. A., Srivastava, H. M. and Trujillo, J. J. Theory and applications of fractional differential equations. North-Holland Mathematics Studies, 204. Elsevier Science B.V., Amsterdam, 2006.

[14] Khoshnevisan, D. Analysis of stochastic partial differential equations. CBMS Regional Conference Series in Mathematics, 119. Published for the Conference Board of the Mathematical Sciences, Washington, DC; by the American Mathematical Society, Providence, RI, 2014. viii+116 pp.

[15] Li, W. V., Shao, Q.-M. Gaussian processes: inequalities, small ball probabilities and applications. Stochastic processes: theory and methods, Handbook of Statist. 19, NorthHolland, Amsterdam, 2001.

[16] Nualart, D. The Malliavin calculus and related topics. Second edition. Probability and its Applications (New York). Springer-Verlag, Berlin, 2006. xiv+382 pp. 
[17] Pipiras, V., Taqqu, M. Integration questions related to fractional Brownian motion. Probab. Theory Related Fields 118 (2000), no. 2, 251-291.

Yaozhong Hu, Jingyu Huang, Khoa Lê and David Nualart: Department of Mathematics, University of Kansas, 405 Snow Hall, Lawrence, Kansas, 66044, USA.

E-mail address: yhu@ku.edu,jhuang@math.utah.edu, khoa.le@ucalgary.ca, nualart@ku.edu

Samy Tindel: Department of Mathematics, Purdue University, West Lafayette, IN 47907, USA.

E-mail address: stindel@purdue.edu 